ARTICLE

\title{
Dichroic spin-valley photocurrent in monolayer molybdenum disulphide
}

Mustafa Eginligil ${ }^{1}$, Bingchen Cao ${ }^{1}$, Zilong Wang ${ }^{1,2}$, Xiaonan Shen ${ }^{1}$, Chunxiao Cong ${ }^{1}$ Jingzhi Shang ${ }^{1}$, Cesare Soci ${ }^{1,2} \&$ Ting $\mathrm{Yu}^{1,3,4}$

The aim of valleytronics is to exploit confinement of charge carriers in local valleys of the energy bands of semiconductors as an additional degree of freedom in optoelectronic devices. Thanks to strong direct excitonic transitions in spin-coupled $K$ valleys, monolayer molybdenum disulphide is a rapidly emerging valleytronic material, with high valley polarization in photoluminescence. Here we elucidate the excitonic physics of this material by light helicity-dependent photocurrent studies of phototransistors. We demonstrate that large photocurrent dichroism (up to 60\%) can also be achieved in high-quality molybdenum disulphide monolayers grown by chemical vapour deposition, due to the circular photogalvanic effect on resonant excitations. This opens up new opportunities for valleytonic applications in which selective control of spin-valley-coupled photocurrents can be used to implement polarization-sensitive light-detection schemes or integrated spintronic devices, as well as biochemical sensors operating at visible frequencies.

\footnotetext{
${ }^{1}$ School of Physical and Mathematical Sciences, Physics and Applied Physics, Nanyang Technological University, 21 Nanyang Link, Singapore 637371, Singapore. ${ }^{2}$ Centre for Disruptive Photonic Technologies, Nanyang Technological University, 21 Nanyang Link, Singapore 637371, Singapore. ${ }^{3}$ Department of Physics, Faculty of Science, National University of Singapore, 2 Science Drive 3, Singapore 117551, Singapore. ${ }^{4}$ Centre for Advanced 2D Materials and Graphene Research Centre, National University of Singapore, 6 Science Drive 2, Singapore 117546, Singapore. Correspondence and requests for materials should be addressed to T.Y. (email: yuting@ntu.edu.sg).
} 
$\mathrm{R}$ esearch on two-dimensional (2D) materials synthesis and characterization has accelerated since the discovery of graphene $^{1}$, for the purpose of utilizing these multifunctional materials in highly efficient nano- and biotechnological platforms ${ }^{2}$. Among 2D materials, particularly transition metal dichalcogenide (TMD) monolayers ${ }^{3,4}$ have shown various interesting properties such as charge density waves $^{5}$, unusual strain ${ }^{6}$ and thermal energy dependence ${ }^{7}$, tunable light emission by chemical control ${ }^{8}$ and fast photoresponse ${ }^{9}$, which can be used in interdisciplinary device applications. Today's nanodevices mostly use charge and spin degree of freedom; however, it is also possible to exploit the valley degree of freedom, which is simply the confinement of charge carriers in momentum space of 2D materials. For instance, in molybdenum disulphide $\left(\mathrm{MoS}_{2}\right)$-a 2D semiconductor TMD and a noncentrosymmertic crystal-inherent broken inversion symmetry in monolayers leads to a large spin-orbit interaction (SOI) that splits the valence band (VB) by $150 \mathrm{meV}^{10}$ and gives rise to strong excitonic transitions due to the direct band gap at low energy $K$ and $-K$ valleys. The upper (lower) VB is associated with the $A(B)$ excitons. The same broken inversion symmetry together with time reversal symmetry is responsible for spin-valley coupling in monolayer $\mathrm{MoS}_{2}$ and similar TMDs $\left(\mathrm{WS}_{2}, \mathrm{WSe}_{2}\right.$ and $\left.\mathrm{MoSe}_{2}\right)$; that is, the sign of the hole spin in the upper (or lower) VB will oppose in different valleys. The descriptive Hamiltonian ${ }^{10}$ can be simply expressed as $\hat{H}=\left[a t\left(\tau k_{x} \hat{\sigma}_{x}+k_{y} \hat{\sigma}_{y}\right)\right]+\left[(\Delta / 2) \hat{\sigma}_{z}\right]+\left[2 \lambda \tau\left(\left(\hat{\sigma}_{z}-1\right) / 4 \hat{s}_{z}\right)\right]$ where $a$ is the lattice constant, $t$ the effective hopping integral, $\tau( \pm 1)$ the valley index, $\hat{\sigma}$ the Pauli matrices, $\Delta$ the energy gap, $2 \lambda$ the spin splitting at the VB, and $\hat{s}_{z}$ the Pauli matrix for spin; $k_{x}$, $k_{y}, k_{z}$ are the momentum components in $x, y, z$ coordinates, respectively. The first term is related to the valley related hopping, the second term is purely spin dependent and the last term describes the spin-dependent phenomena as a result of SOI in each valleys. The spin-valley coupling in the last term, which leads to the valley polarization, was demonstrated experimentally by light helicity-dependent photoluminescence measurements corresponding to the $A$ and $B$ excitons in monolayer $\mathrm{MoS}_{2}{ }^{11,12}$. Recently, in suspended monolayer $\mathrm{MoS}_{2}$ samples, photocurrent (PC) due to the $A$ and $B$ excitons was also revealed ${ }^{13}$. A useful framework to study light-matter interactions in $2 \mathrm{D}$ materials is based on PC experiments performed by circularly polarized excitation $^{14}$. However, helicity-dependent PCs due to excitons with on-resonance and off-resonance excitations in monolayer TMDs are still to be uncovered.

There have been extensive studies on growth techniques of TMDs to synthesize high-quality, single-crystal and large-area samples. Among these efforts, high-quality single layer but small area $(<10 \mu \mathrm{m})$ samples that are mechanically cleaved, have shown strong photoluminescence, fluorescence ${ }^{15,16}$, high-mobility-lowpower switching ${ }^{17}$, and large photoresponse ${ }^{18}$. Alternatively, samples grown by chemical vapour deposition (CVD) methods ${ }^{19-}$ 22 also yield quite intense light emission ${ }^{23}$, particularly monolayers of tungsten disulphide $\left(\mathrm{WS}_{2}\right)$ have shown stronger photoluminescence than mechanically cleaved monolayers ${ }^{24}$. In terms of electrical transport, both mechanically cleaved and CVD grown $\mathrm{WS}_{2}$ and $\mathrm{MoS}_{2}$ have $n$-type behaviour. In addition, since CVD method allows also growing multiple 2D materials to form hetero- and hybrid structures ${ }^{4}$, it offers to tailor functional physical systems and, particularly for optoelectronics and spin-dependent valleytronics. The possibility of synthesizing high-quality samples with much larger size $(>500 \mu \mathrm{m})$ than mechanically cleaved samples gives CVD growth techniques a major advantage in the development of future technologies based on TMDs.

In this work, we demonstrate spin-coupled valley-dependent dichroic PC of a CVD grown single-layer $\mathrm{MoS}_{2}$ phototransistor excited by on-resonance and off-resonance photon energies (1.96 and $2.33 \mathrm{eV}$, respectively). A PC due to the circular photogalvanic effect (CPGE) arises as a result of circularly polarized light incident on monolayer $\mathrm{MoS}_{2}$ with an oblique angle. The spin-valley coupling, the valley selection rules and the excitation frequencies determine the magnitude of PC for left and right circularly polarized states. For excitations that are on-resonance with the excitons, there should be a difference between the CPGE PCs due to left and right circularly polarized excitations; while for excitations, which are off-resonance with the excitons, there should be no difference between these CPGE PCs. Our PC measurements evidence this variation and reveal a high-CPGE PC polarization. We remark on further possibilities of making use of this unique opto-valleytronic control for interdisciplinary applications.

\section{Results}

Spin-valley coupling and circular photogalvanic effect. The phenomenological expression of light helicity-dependent PC is strictly determined by the crystal symmetry, the angle of incidence, $\theta$, (with respect to the normal to the sample plane), the azimuthal angle, $\phi$, (light propagation with respect to the $x$-direction in the $x-y$ plane) of the excitation beam and the angle of photon polarization, $\varphi$, (with respect to the electric field vector direction) ${ }^{14}$. In transverse geometry (as shown in the experimental configuration) in which light is directed in the $x$ $z$ plane and the PC is measured in the $y$-direction, $\phi$ is fixed. If not otherwise specified, in this work, we used $\phi=90^{\circ}$ and $\theta=45^{\circ}$, so that by rotating a quarter-wave plate (QWP), polarization of a linearly polarized laser can be varied from $0^{\circ}$ linear $(\leftrightarrow)$ to $45^{\circ}$ left circular $(\sigma-)$, back to $90^{\circ}$ linear $(\leftrightarrow)$ to $135^{\circ}$ right circular $(\sigma+)$ and then back to $180^{\circ}$ linear $(\leftrightarrow)$ polarization, and so on.

Monolayer $\mathrm{MoS}_{2}$ has $D_{3 h}^{1}$ crystal symmetry ${ }^{10-12}$. When a Mo atom is in an inversion centre, there is no coinciding atom when a $\mathrm{S}$ atom is projected to the $2 \mathrm{D}$ plane. When such a symmetry system is subjected to a circularly polarized light field with an oblique angle $\theta,\left(\theta \neq 0^{\circ}\right)$ a $\mathrm{PC}$ with $\sin 2 \varphi$ dependence-current due to the CPGE, $j_{\mathrm{CPGE}}$ will be the major PC. The angle of incidence $\theta$ dependence of $j_{\mathrm{CPGE}}$ is $\sin \theta$. At the same time, a PC with $\cos 4 \varphi$ dependence, which is due to the transfer of light momentum to electrons, is also present-the so-called linear photon drag effect (LPDE), $j_{\text {LPDE }} 14$. In addition, a PC similar to $j_{\mathrm{CPGE}}$ in origin, but related to the linear polarization of light as $j_{\mathrm{LPDE}}$-linear photogalvanic effect (LPGE), $j_{\mathrm{LPGE}}{ }^{14}$ is also possible, although it should ideally vanish at $\sigma+$ and $\sigma-$ excitations. On the basis of these three contributions, the total PC can be generally expressed by the phenomenological formula $J=C_{1} \sin 2 \varphi+L_{1} \sin 4 \varphi+L_{2} \cos 4 \varphi+D$. Here $C_{1}$ is the coefficient for the CPGE current $\left(j_{\mathrm{CPGE}}=C_{1} \sin 2 \varphi\right) ; L_{1}$ for the LPGE current $\left(j_{\mathrm{LPGE}}=L_{1} \sin 4 \varphi\right) ; L_{2}$ for the LPDE current $\left(j_{\mathrm{LPDE}}=L_{2} \cos 4 \varphi\right)$; and $D$ is polarization-independent term.

In a recent work on multilayer $\mathrm{WSe}_{2}$, the $j_{\mathrm{CPGE}}$ was observed due to absorption at $\Lambda$ valleys-intraband transitions within the conduction band $(\mathrm{CB})^{25}$. In the case of monolayer $\mathrm{MoS}_{2}$, spinvalley coupling will be at $K$ valleys and the $j_{\mathrm{CPGE}}$ should be due to interband transitions from the VB to the $\mathrm{CB}$, for circularly polarized radiation with energies closer to the exciton energies. When spin couples to valley ${ }^{10}$, sign of the spin states of $A$ (or $B$ ) excitons in one valley oppose to that in the other valley. By a simple approach, when excited by $\sigma+(\sigma-)$ excitations with energy slightly higher than the formation/dissociation energy of $A$ exciton, an electron in VB makes an interband transition in $K$ $(-K)$ valley, with hole spin up $\uparrow$ (spin down $\downarrow$ ). Similarly, when excited by $\sigma+(\sigma-)$ excitations with energy slightly higher than 
the formation/dissociation energy of $B$ exciton, an electron in VB makes an interband transition in $K(-K)$ valley, with hole $\downarrow(\uparrow)$ (Fig. 1). This coupling can be understood by the PC generated when circularly polarized light-field irradiated on the sample is perpendicular to the electrodes (as shown in the experimental setup). In such a case, the velocities $v_{v}$ of the carriers in the VB, which absorb this light field, will have a contribution additional to the one due to the band dispersion $\delta \varepsilon(\mathbf{k}) / \delta \mathbf{k}$ for energy $\varepsilon(\mathbf{k})$ in $\mathbf{k}$ space. That contribution is the anomalous velocity which appears as the second term in $\boldsymbol{v}_{\mathbf{v}}(\mathbf{k})=(1 / \hbar)(\delta \varepsilon(\mathbf{k}) / \delta \mathbf{k})-((e / \hbar) \mathbf{E} \times \mathbf{\Omega})^{26}$, where $\hbar$ is the Planck constant, $\mathbf{E}$ the electric field vector, and $\boldsymbol{\Omega}$ the Berry curvature that has the same magnitude but different sign in $K$ and $-K$ valleys, $\Omega_{K}(-K)=+(-) \Omega \hat{z}$. Then, for excitation that is on-resonance with the $A$ excitons, we can write the magnitude of $\mathbf{v}_{\mathbf{v}}(\mathbf{k})$ at $K(-K)$, which actually depends on the light helicity, as $v_{v(i) \uparrow(\downarrow)}=\left|(e / \hbar) \mathbf{E} \times \boldsymbol{\Omega}_{K(-K)}\right|$; and for

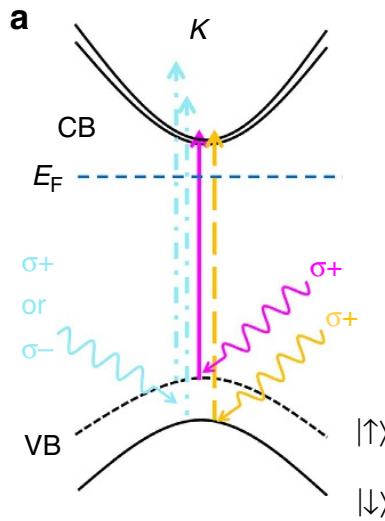

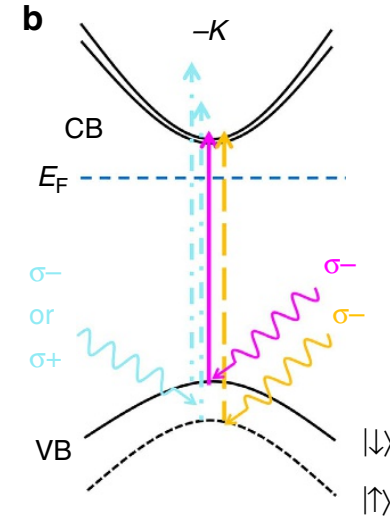

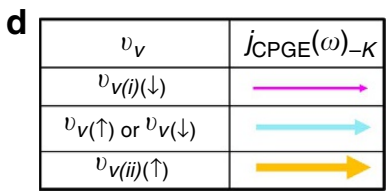

Figure 1 | Band diagram of $K$ and $-K$ valleys of $\mathbf{M o S}_{2}$ and photocurrent generation. The $\sigma+$ and $\sigma-$ excitations which are on-resonance with $A$ exciton-a hole in the upper valence band (VB) (black solid (dash) curve in a.b for $K(-K)$ valley) and electron in the conduction band (CB) (black solid curves in $\mathbf{a}, \mathbf{b}$ ) lead to photocurrent (PC) generation in $K$ and $-K$ valleys, respectively. Similarly, the $\sigma+$ and $\sigma-$ excitations which are on-resonance with $B$ exciton-a hole in the lower VB (black solid (dash) curve in $\mathbf{a}, \mathbf{b}$ for $K(-K)$ valley) and electron in the CB lead to PC generation. The blue horizontal dashed lines in $\mathbf{a}, \mathbf{b}$ are the Fermi level $E_{F}$ which can be tuned by $V_{g}$. The VBs are split by $150 \mathrm{meV}$, while the splitting in the CB is negligible. The purple vertical solid (orange long dash) arrows show interband transitions associated with the $A(B)$ excitons when illuminated by on-resonance laser; while turquoise vertical dash-dot arrows show possible transitions by off-resonance laser. For on-resonance laser, $\sigma+(\sigma-)$ excitation in $K(-K)$ valley is linked with hole spin $\uparrow(\downarrow)$ due to the spin-valley coupling for $A$ excitons. Thus based on theory, the circular photogalvanic current in $K$ valley, $j_{\text {CPGE }}(\omega)_{K}$ due to the $v_{v(i) \uparrow}$ at $\sigma+$ excitation will be larger (shown by thicker purple right arrow in c as a result of transitions represented by purple vertical solid arrows in a) than the $j_{\mathrm{CPGE}}(\omega)_{-K}$ due to the $v_{v(i) \downarrow}$ at $\sigma-$ excitation (shown by thinner purple right arrow in $\mathbf{d}$ as a result of transitions represented by purple vertical solid arrows in $\mathbf{b}$ ), which is the reason of the pronounced photocurrent polarization. Similar but opposite scenario for $v_{v(i i) \downarrow}$ and $v_{v(i i) \uparrow}$, by exchanging valleys and spins applies for $B$ excitons (orange right arrows). For off-resonance laser, $\sigma+$ and $\sigma-$ excitations yield a negligible photocurrent polarization due to simultaneous populations of both valleys, as shown by equivalent turquoise right arrows in both $\mathbf{c}, \mathbf{d}$. excitation which is on-resonance with the $B$ excitons, as $v_{v(i i) \downarrow(\uparrow)}=\left|(e / \hbar) \mathbf{E} \times \boldsymbol{\Omega}_{K(-K)}\right|$. In this case, the PC due to the $v_{v(i) \uparrow}\left(v_{v(i i) \downarrow}\right)$, at $\sigma+$ excitation will be larger than the PC due to the $v_{v(i) \downarrow}\left(v_{v(i i) \uparrow}\right)$ at $\sigma-$ excitation. As an example, for the $A$ excitons, the CPGE current $j_{\mathrm{CPGE}}(\hbar \omega)$, ( $\omega$ is the excitation frequency) is given by $j_{\mathrm{CPGE}}(\omega)_{\sigma+(\sigma-)}$ or $j_{\mathrm{CPGE}}(\omega)_{K(-K)}=(8 e \pi / \hbar)\left[\sum_{v(i) \rightarrow c}\right.$ $\left(v_{c} \tau\left(\varepsilon_{c}\right)-\left(\left(\delta \varepsilon_{v(i)} / \delta k\right)-v_{v(i) \uparrow(\downarrow)} \tau\left(\varepsilon_{v(i)}\right)\right)\right] \quad\left|M_{v(i) \rightarrow c}\right| \quad\left|f\left(\varepsilon_{c}\right)-f\left(\varepsilon_{v(i)}\right)\right|$ $\delta\left(\varepsilon_{c}-\varepsilon_{v(i)}-\hbar \omega\right)$, and $\left(v_{c} \tau\left(\varepsilon_{c}\right)\right)>\left(\left(\delta \varepsilon_{v(i)} / \delta k\right)-v_{v(i) \uparrow(\downarrow)} \tau\left(\varepsilon_{v(i)}\right)\right)$; where $v(i)$ is the initial VB state on-resonance with the $A$ excitons and $c$ is final $\mathrm{CB}$ states, $M_{v \rightarrow c}$ is the transition matrix from the upper $\mathrm{VB}$ states to $\mathrm{CB}$ states, $v_{c}$ is the electron velocity in the $\mathrm{CB}$ states, $\tau\left(\varepsilon_{v}\right)$ and $\tau\left(\varepsilon_{c}\right)$ are the momentum relaxation times in the $\mathrm{VB}$ and $\mathrm{CB}$, respectively. The Fermi-Dirac functions $f\left(\varepsilon_{c}\right)$ and $f\left(\varepsilon_{v}\right)$ are dependent on the gate voltage and to study purely interband transitions, the Fermi level must be lowered by negative gate voltage (off state). Here we remark at the condition $\left(v_{c} \tau\left(\varepsilon_{c}\right)\right)>\left(\left(\delta \varepsilon_{\mathrm{v}} / \delta k\right)-v_{v \uparrow(\downarrow)} \tau\left(\varepsilon_{v}\right)\right)$, which determines the sign of $j_{\mathrm{CPGE}}(\omega)_{K(-K)}$. We can consider the $j_{\mathrm{CPGE}}(\omega)_{K(-K)}$ with three components: (1) $\propto v_{c} \tau\left(\varepsilon_{c}\right) ;(2) \propto\left(\delta \varepsilon_{v} / \delta k\right)$; and (3) $\propto v_{v(i) \uparrow(\downarrow)} \tau\left(\varepsilon_{v(i)}\right)$. The third part (anomalous part) is the one that differs for on-resonance excitations. Anomalous parts of the electron velocities in the upper $\mathrm{VB}$ states for $K$ and $-K$ valleys for excitations on-resonance with the $A$ excitons are $v_{v(i) \uparrow}$ and $v_{v(i) \downarrow}$, respectively, which are equal in magnitude but opposite in sign. For excitations on-resonance with the $B$ excitons, anomalous parts of the electron velocities in the lower VB states for $K$ and $-K$ valleys are $v_{v(i i) \uparrow}$ and $v_{v(i i) \downarrow}$, respectively. On the basis of valley selection rules, the sign of $v_{v(i) \uparrow(\downarrow)}$ opposes with the sign of $v_{v(i i) \uparrow(\downarrow)}$, leading to a PC with opposite sign. However, the overall PC $j_{\mathrm{CPGE}}(\omega)_{K}$ and $j_{\mathrm{CPGE}}(\omega)_{-K}$ should be in the same direction as shown in upper panel of Fig. 1, because $\left(v_{c} \tau\left(\varepsilon_{c}\right)\right)>\left(\left(\delta \varepsilon_{v} / \delta k\right)-\right.$ $\left.v_{v \uparrow(\downarrow)} \tau\left(\varepsilon_{v}\right)\right)$. Hence on $\sigma+(\sigma-)$ excitations on-resonance with these excitons, the PC $j_{\mathrm{CPGE}}(\omega)_{\sigma+(\sigma-)}$ will be determined by the contributions due to $v_{v(i) \uparrow(\downarrow)}$ and $v_{v(i i) \uparrow(\downarrow)}$. This will make the main difference between $j_{\mathrm{CPGE}}(\omega)_{K}$ and $j_{\mathrm{CPGE}}(\omega)_{-K}$. Therefore, spin-coupled valley-dependent currents are expected due to excitons formed after light helicity-selective transitions of electrons from the VB to the CB. For off-resonance excitations, the PC expression due to the CPGE is more complicated; $j_{\mathrm{CPGE}}(\omega)_{\sigma+(\sigma-)}$ can be $j_{\mathrm{CPGE}}(\omega)_{K}$ or $-K(-K$ or $K)=(8 e \pi / \hbar)$ $\left[\sum_{v \rightarrow c}\left(v_{c} \tau\left(\varepsilon_{c}\right)\right)-\left(\left(\delta \varepsilon_{v} / \delta k\right)-v_{v(\uparrow \text { or } \downarrow)} \tau\left(\varepsilon_{v}\right)\right)\right] \quad\left|M_{v \rightarrow c}\right|\left|f\left(\varepsilon_{c}\right)-f\left(\varepsilon_{v}\right)\right|$ $\delta\left(\varepsilon_{c}-\varepsilon_{v}-\hbar \omega\right)$, in which $v$ can be $v(i)$ (upper VB) or $v(i i)$ (lower $\mathrm{VB})$. However, we can simply expect that for off-resonance excitation, $j_{\mathrm{CPGE}}(\omega)_{\sigma+}$ and $j_{\mathrm{CPGE}}(\omega)_{\sigma-}$ will yield similar current values, since the off-resonance excitation simultaneously populates both $K$ and $-K$ valleys ${ }^{11}$. In the off-resonance excitation, we cannot take advantage of the valley selection rules and spin-valley coupling, which is reflected in the variance between $j_{\mathrm{CPGE}}(\omega)_{\sigma+}$ and $j_{\mathrm{CPGE}}(\omega)_{\sigma-}$ in on-resonance excitations. This underlines the importance of strong excitonic character that can be obtained in high-quality samples to observe a pronounced difference between $j_{\mathrm{CPGE}}(\omega)_{\sigma}+$ and $j_{\mathrm{CPGE}}(\omega)_{\sigma-}$.

Device characterization and photocurrent. Figure 2 shows the microphotoluminescence of the device prepared by CVD as shown in the inset. The microphotoluminescence measurement was performed by $532-\mathrm{nm}$ laser excitation at room temperature. The strong excitonic peak of $A(B)$ exciton corresponds to pair of an electron in the $\mathrm{CB}$ and a hole in the upper (lower) band of the split VB at $K$ valleys. The PL peak for $A(B)$ excitons in these samples is around $1.84 \mathrm{eV}(\sim 2 \mathrm{eV})$ and the $A$ excitons are much stronger in intensity. The quality of the flakes was demonstrated by Raman microspectroscopy and contrast-enhanced fluorescence optical microscope image in Supplementary Figs 1 and 2, respectively. Initial electrical measurements of monolayer $\mathrm{MoS}_{2}$ field-effect transistors were performed in dark. Drain current 


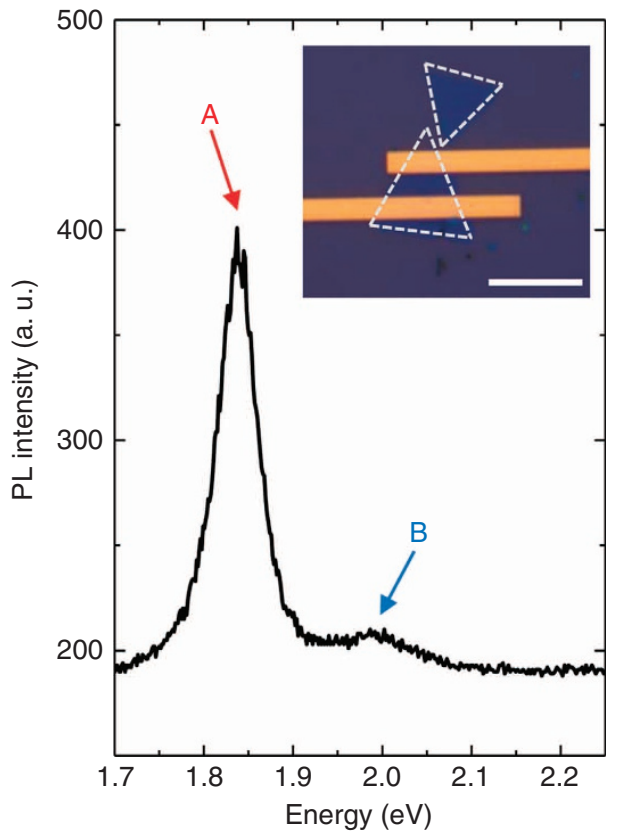

Figure 2 | Photoluminescence of the sample. The strong excitonic $A$ peak is at $1.84 \mathrm{eV}$. The other excitonic $B$ peak is at $2 \mathrm{eV}$. The respective optical image is shown in the inset. The dash lines in the inset are drawn to make easier to visualize the flakes. The orange bars are Ti/Au contacts. Scale bar, $5 \mu \mathrm{m}$.

versus drain voltage $\left(V_{\mathrm{d}}\right)$ characteristics, at zero gate voltage $\left(V_{\mathrm{g}}\right)$, are shown in Supplementary Fig. 3. For $10 \mathrm{~V}$ applied $V_{\mathrm{d}}$, the fieldeffect transistors characteristics mark an on-state at $-30 \mathrm{~V}$ (Fig. 3a). By a linear fit between 30 and $40 \mathrm{~V}$, we can estimate the mobility of these devices from $\mu=(L / W)(\mathrm{d} I / \mathrm{d} V)\left(1 / C V_{\mathrm{d}}\right)$. Here $L$ is the device length, $W$ the device width and $C$ is the back gate capacitance. The mobility is $\sim 0.5 \mathrm{~cm}^{2} \mathrm{~V}^{-1} \mathrm{~s}-1$, as shown in Supplementary Fig. 4, which is in agreement with the reported values ${ }^{18}$. Subsequent drain current measurements on illumination by $2.33 \mathrm{eV}$ laser with $20 \mathrm{~mW} \mathrm{~cm}^{-2}$ power density, and comparison with the dark measurements, are illustrated as a function of $V_{\mathrm{d}}$ in Supplementary Fig. 5. The drain current as a function of $V_{\mathrm{g}}$, on illumination $\left(I_{\text {Illumination }}\right)$ and in dark $\left(I_{\text {Dark }}\right)$ are compared in Fig. 3a. The ratio of $I_{\text {Illumination }} / I_{\text {Dark }}$ is $\sim 10^{4}$ in the off state $\left(V_{\mathrm{g}}=-35 \mathrm{~V}\right)$.

Laser power density dependence of drain current at $-40 \mathrm{~V} V_{\mathrm{g}}$ and $10 \mathrm{~V} V_{\mathrm{d}}$ was measured. Figure 3 shows PC (Illumination$\left.I_{\text {Dark }}\right)$ versus laser power density. Photoresponsivity $(R)$ at the minimum power for the device shown in the inset to Fig. 2 is about $3.5 \mathrm{~A} \mathrm{~W}^{-1}$, which is about two orders of magnitude lower than the reported one $\mathrm{e}^{18}$. The power dependence of the measured

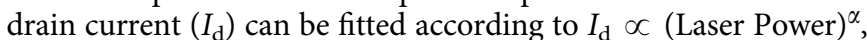
where $\alpha$ is about 0.7 for our samples and the reported $\mathrm{MoS}_{2}{ }^{18}$. At higher power densities, $R$ drops either due to the trap states in $\mathrm{MoS}_{2}$ or the interface of $\mathrm{MoS}_{2}$ and the substrate ${ }^{27}$. Therefore, to elucidate the photoinduced transport effects of monolayer $\mathrm{MoS}_{2}$, further PC measurements at both high and low laser power densities were performed using conventional amplitude modulation and lock-in detection.

In Fig. 4b, gate dependence of PC signal for different $V_{\mathrm{d}}$ and laser power density with $2.33-\mathrm{eV}$ excitation is presented for $\varphi=0^{\circ}$ and $\theta=45^{\circ}$ configuration as described in the experimental setup of Fig. 4a. Both PC signal amplitude and phase were measured. Phase is around $0^{\circ}$ while the $V_{\mathrm{g}}, V_{\mathrm{d}}$ and laser power were varied; no sign change of $\mathrm{PC}$ was observed as shown in Supplementary Fig. 6. At high $V_{\mathrm{d}}$ of $10 \mathrm{~V}$, both
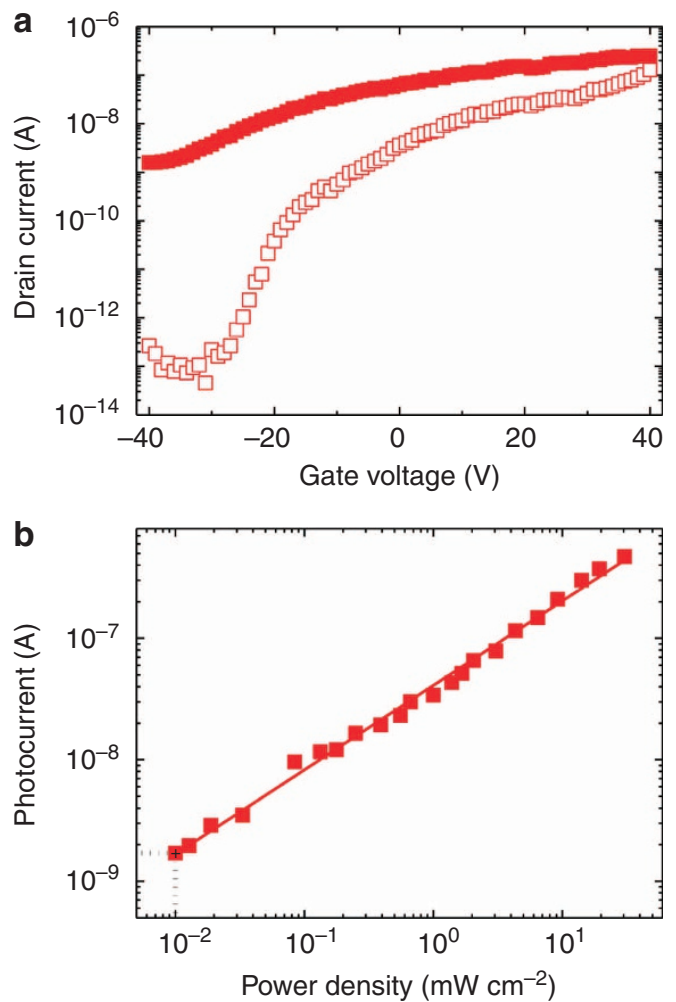

Figure 3 | Device characterization of the sample. (a) Gate voltage dependence of drain current, at $10 \mathrm{~V}$ drain voltage, in dark (empty squares) and upon $20 \mathrm{~mW} \mathrm{~cm}^{-2}$ illumination by $2.33 \mathrm{eV}$ laser (full squares). The ratio of $I_{\text {Illumination }}$ to $I_{\text {Dark }}$ is about $10^{4}$ in the off state $\left(V_{g}=-35 \mathrm{~V}\right)$ (b) Photocurrent measured as a function of laser power density at $V_{g}=-40 \mathrm{~V}$ is shown. The data (full squares) are fit to a straight line and a photoresponsivity $(R)$ of $3.5 \mathrm{AW}^{-1}$ is obtained.

high-power-density data $\left(9.78 \mathrm{~W} \mathrm{~cm}^{-2}\right)$ and low-power-density data $\left(60 \mathrm{~mW} \mathrm{~cm}^{-2}\right)$ are being affected by bolometric effects as in the AB-stacked bilayer graphene ${ }^{28}$, which are due to the high channel doping. The PC amplitude increases almost linearly from 100 to $200 \mathrm{nA}$ from $-40 \mathrm{~V}$ to $40 \mathrm{~V}$ for high power density; from 3 to $20 \mathrm{nA}$ for low power density. At low $V_{\mathrm{d}}$ of $0.7 \mathrm{~V}$ and low power density $\left(60 \mathrm{~mW} \mathrm{~cm}^{-2}\right)$, the measured $\mathrm{PC}$ is dominated by photogalvanic effect, photon drag effect and low channel doping. The PC amplitude increases logarithmically from 0.1 to $8 \mathrm{nA}$, in accordance with the gate dependence of drain current data in Fig. 4b. Low $V_{\mathrm{d}}$ also prevents from artificial PC such as photogating effects ${ }^{29}$ and photothermal effects ${ }^{30}$. Therefore, intrinsic photogalvanic effects are expected to appear at low $V_{\mathrm{d}}$ and low illumination intensity. Here we focus on this regime to address subtle effects of light helicity on spin-valley PCs, which has been a subject of fundamental opto-spintronic applications ${ }^{31-33}$.

Helicity-dependent PC by 2.33 and $1.96 \mathrm{eV}$ excitations. Figure 5 shows our light helicity-dependent PC results by varying the angle of photon polarization $(\varphi)$ for off-resonance $(2.33 \mathrm{eV}$ photon energy, $0.7 \mathrm{~V}$ drain voltage and $60 \mathrm{~mW} \mathrm{~cm}^{-2}$ illumination intensity) and for on-resonance excitation $(1.96 \mathrm{eV}$ photon energy, $2 \mathrm{~V}$ drain voltage and $100 \mathrm{~mW} \mathrm{~cm}^{-2}$ illumination intensity) at $V_{\mathrm{g}}=-40 \mathrm{~V}$. As expected, for off-resonance excitation, (Fig. 5a) the PC exhibits no clear dependence on angle of photon polarization (that is, $\sigma+$ and $\sigma-$ excitation). Indeed, there is no preferential optical transition (due to electron-photon interaction and scattering events) from the upper or lower VBs to 
a

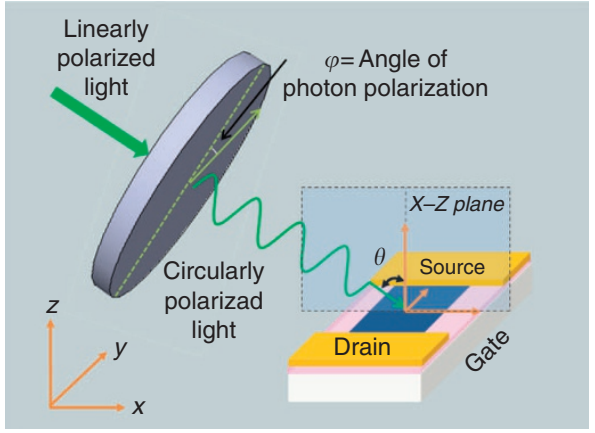

b

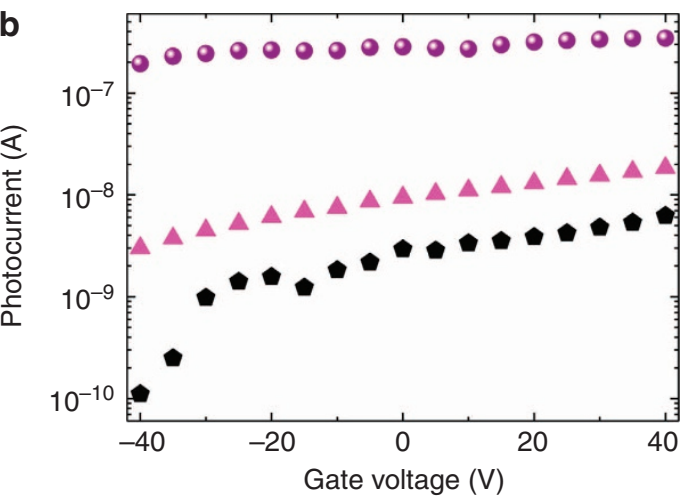

Figure 4 | Experimental configuration and photocurrent. (a) Description of photocurrent setup using the lock-in technique to collect the photocurrent (PC) amplitude and phase is given. Using a linearly polarized laser with a quarter-wave plate (QWP), circularly polarized light obtained and directed on the sample with an oblique angle $\theta$. By rotating QWP it is possible to change the angle of photon polarization $\varphi$. (b) PC of the sample illuminated by $2.33 \mathrm{eV}$ laser, for $\varphi=0^{\circ}$ and $\theta=45^{\circ}$. Spheres are the data for $10 \mathrm{~V}$ drain voltage and $9.78 \mathrm{~W} \mathrm{~cm}^{-2}$ laser power density. Up triangles are the data for $10 \mathrm{~V}$ drain voltage and $60 \mathrm{~mW} \mathrm{~cm}^{-2}$. Pentagons are the data for $0.7 \mathrm{~V}$ drain voltage and $60 \mathrm{~mW} \mathrm{~cm}^{-2}$. Helicity-dependent $P C$ was performed in this regime (off state) in which there are only photogalvanic and photon drag effects.

the $\mathrm{CB}$, in terms of valleys, on absorption of photon energies off-resonance with the excitonic transition. The data are fitted to the phenomenological formula $J=C_{1} \sin 2 \varphi+L_{1} \sin 4 \varphi+L_{2}$ $\cos 4 \varphi+D$ and the fitting parameters are tabulated in Table 1 . The component of PC response related to circularly polarized light is the $j_{\text {CPGE }}$. But for this excitation, $j_{\mathrm{LPDE}}$ is the dominant contribution. Thus, by subtracting $j_{\mathrm{LPDE}}$ and polarizationindependent term from the measured PC, PC $(\sigma \pm)$, one can obtain $j_{\mathrm{CPGE}}(\sigma \pm)=\mathrm{PC}(\sigma \pm)-\left(-L_{2}+D\right)$. Then PC polarization can be defined as $P=\left[\left|j_{\mathrm{CPGE}}(\sigma+)\right|-\left|j_{\mathrm{CPGE}}(\sigma-)\right|\right] /\left[\mid j_{\mathrm{CPGE}}\right.$ $\left.(\sigma+)|+| j_{\mathrm{CPGE}}(\sigma-) \mid\right]$. By using the parameters in Table $1, P$ is $0.8 \pm 0.4 \%$. On the basis of microscopic description of the CPGE valley current, $v_{v(i)^{-}}$and $v_{v(i i)}$-dependent terms cancel each other and eventually results in a negligible PC polarization.

The picture changes markedly in the case of $\mathrm{PC}$ observed for excitation with photon energy $=1.96 \mathrm{eV}$, which is on-resonance with the excitonic transitions. This photon energy is on-resonance mainly with $A$ excitons, as explained in Supplementary Note 1 . The angle of photon polarization dependence of PC exhibits a clear difference between $\sigma+$ and $\sigma-$ excitations in Fig. 5b, as expected for spin-coupled valley currents with resonant excitation. As shown in Table 1, the parameter $C_{1}$ is much larger compared with the $2.33-\mathrm{eV}$ excitation, although $L_{1}$ still small and $L_{2}$ gets relatively smaller. The coefficients $C_{1}$ and $L_{2}$ stand as the most prominent ones in
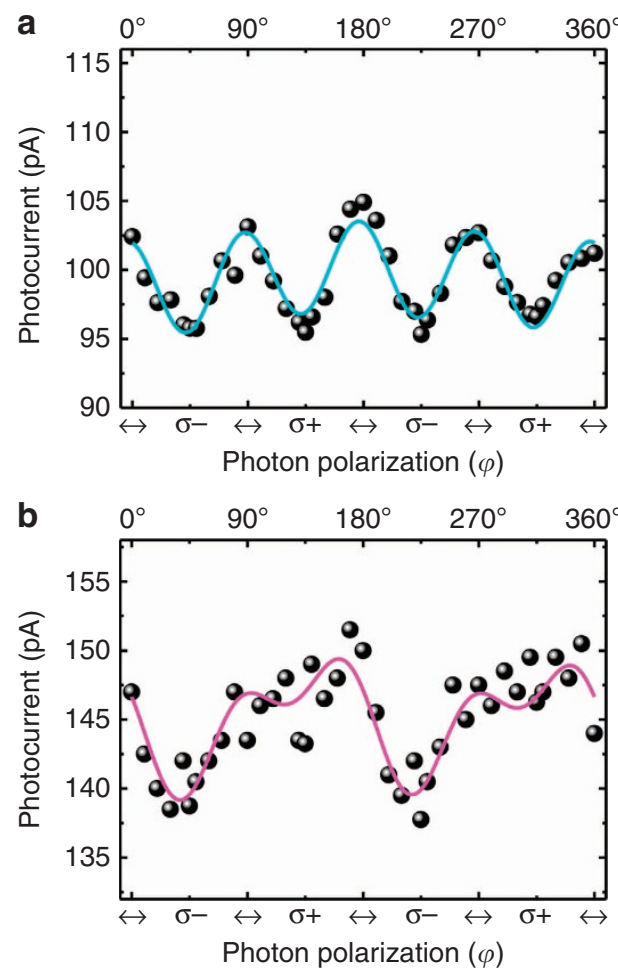

Figure 5 | Spin-coupled valley-dependent dichroic photocurrent in monolayer $\mathbf{M o S}_{\mathbf{2}}$. (a) Photocurrent (PC) as a function of angle of photon polarization $\varphi$ when illuminated by $2.33 \mathrm{eV}$ (off-resonance with the excitons) laser is shown. The PC data are collected by rotating a quarter-wave plate which changes polarization of a linearly polarized laser from $0^{\circ}$ linear $(\leftrightarrow)$ to $45^{\circ}$ left circular $(\sigma-)$, back to $90^{\circ}$ linear $(\leftrightarrow)$ to $135^{\circ}$ right circular $(\sigma+)$, and then back to $180^{\circ}$ linear $(\leftrightarrow)$ polarization, and so on. The (cyan) curve is the fitting function based on the phenomenological PC formula for monolayer $\mathrm{MoS}_{2}$, yielding negligible polarization between $\sigma+$ and $\sigma-$ excitations. (b) PC as a function of $\varphi$ when illuminated by $1.96 \mathrm{eV}$ (on-resonance) laser. The (magenta) curve is the fitting function based on the phenomenological PC formula for monolayer $\mathrm{MoS}_{2}$, yielding a polarization of $\sim 60 \%$ between $\sigma+$ and $\sigma-$ excitations, which control the valley currents in $K$ (linked with hole spin up) and $-K$ (linked with hole spin down) valleys, respectively.

\section{Table 1 | Photocurrent components of the sample excited by circularly polarized light.}

\begin{tabular}{ccccc} 
& $\boldsymbol{C}_{\mathbf{1}}$ & $\boldsymbol{L}_{\mathbf{1}}$ & $\boldsymbol{L}_{\mathbf{2}}$ & $\boldsymbol{D}$ \\
\hline $1.96 \mathrm{eV}$ & -3.5 & -1.5 & 1.8 & 145.1 \\
$2.33 \mathrm{eV}$ & -0.2 & -0.7 & 3.2 & 99.5 \\
\hline
\end{tabular}

Parameters are determined from the phenomenological photocurrent $(P C)$ formula fittings for the monolayer $\mathrm{MoS}_{2}$ grown by chemical vapor deposition. The PC components are obtained by 1.96 and $2.33 \mathrm{eV}$ circularly polarized excitations.

determining polarization-dependent $\operatorname{PC}(\sigma \pm)$. The parameter $C_{1}$ becomes negligible for the on-resonance excitation once the angle of incidence $\theta=0^{\circ}$, as shown in Supplementary Fig. 7 and Supplementary Table 1. The figure of merit for PC polarization can be taken as the ratio of $\left|C_{1}\right| /\left|L_{2}\right|$, which is 0.06 for off-resonance $(2.33 \mathrm{eV})$ excitation and 1.94 for on-resonance $(1.96 \mathrm{eV})$ excitation. In terms of microscopic origin, the PC polarization for the excitation on resonance with the $A$ excitons can be qualitatively given as $\sim\left|v_{v(i)} \tau\left(\varepsilon_{v}\right)\right| /\left|v_{c} \tau\left(\varepsilon_{c}\right)\right|$ based on the $j_{\text {CPGE }}$ defined earlier in Fig. 1 . In other words, $j_{\text {CPGE }}$ dominates and the concomitant $P$ has high values of $60 \pm 30 \%$ when excited 
by $1.96 \mathrm{eV}$. This value is larger when compared with PL polarization at zero $V_{\mathrm{g}}$ presented in Supplementary Fig. 8. This could be due to loss of polarization during the process of circularly polarized excitation and collection in PL measurements. Also, in PC measurements we are able to identify purely the CPGE-dependent valley current and additional effects in PL are suppressed. The large error in $P$ is due to the fluctuations in the data as explained in Supplementary Note 2. It is possible to obtain a better signal by further improvement of device fabrication. As a control experiment, we used the same experimental setup at $\theta=45^{\circ}$ with on-resonance laser, only by replacing circularly polarized light by linearly polarized light (we used half-wave plate instead of QWP); the data at $45^{\circ}, 135^{\circ}, 225^{\circ}$ and $315^{\circ}$ yield similar values, unlike circularly polarized excitation. We also give a comparison of our results of the CVD grown monolayer $\mathrm{MoS}_{2}$ sample with the results of a mechanically cleaved monolayer $\mathrm{MoS}_{2}$ sample in Supplementary Figs 9 and 10, by electrical measurements in Supplementary Figs 3, 4 and 11, photoresponse in Supplementary Fig. 12, circularly polarized PL in Supplementary Fig. 13 and light helicity-dependent spin-valley current in Supplementary Fig. 14 and in Supplementary Table 2. In mechanically cleaved sample, the PC polarization that reflects the spin-valley coupling is relatively smaller than the CVD sample as explained in Supplementary Note 2, which makes CVD grown $\mathrm{MoS}_{2}$ devices promising for spin-dependent optovalleytronic applications.

\section{Discussion}

Our results of exciton-related spin-coupled valley-dependent PC are consistent with helicity-dependent PL measurements ${ }^{11}$ and recent observation of the valley Hall effect in monolayer $\mathrm{MoS}_{2}{ }^{34}$. These results may have a number of applications in nanoeletronics and photonics, such as such as polarizationsensitive light-detection schemes or integrated spintronic devices. Furthermore, more examples can be developed based on magnetoelectric effects of bilayer $\mathrm{TMDs}^{35}$ and spin-orbit interaction in graphene/TMD hybrid systems ${ }^{36}$, since CVD grown TMD devices are promising in terms of electrical transport $^{37}$. Thanks to room-temperature operation, the control over spin-dependent valley PC is more application oriented than the recently discovered helicity-dependent PC of topological insulators $^{38}$. Moreover, special attention should be paid to biological environments, which are sensitive to light handedness and colour. Examples can be given in the area of light-harvesting chlorophyll (LHC) research ${ }^{39}$ and biomolecule-nanoparticle interaction ${ }^{40}$. For instance, light helicitiy dependence has been demonstrated for the chiral LHC, which functions around red colour $(633 \mathrm{~nm}-1.96 \mathrm{eV})$ wavelength but insensitive to green colour $^{41}$. This realizes the possibility of integrating chiral LHC with ultrasensitive spin-valley-coupled $\mathrm{MoS}_{2}$ phototransistors. Also, the biophysical community has been investigating the protein binding to nanomaterials for vision-related nanomedical purposes ${ }^{42}$. On the basis of our observations, we believe it is viable to utilize the unique optoelectronic features of $\mathrm{MoS}_{2}$ in the exploration of light sensitive bio-nano systems.

We emphasize that high-quality monolayer $\mathrm{MoS}_{2}$, which is a spin-valley coupling system grown by chemical vapour deposition fabricated as phototransistor exhibits helicitydependent PC behaviour, based on its unique symmetry conditions. Spin-dependent valley currents can be generated by circularly polarized laser, and can be manipulated by laser excitation and electrical gating. We were able to demonstrate that in $K$ or $-K$ valleys with given spin (up or down, respectively) exciton-related electron conduction can take place, and PC polarization as high as $60 \%$ was obtained by choosing a suitable visible laser with a known circular polarization $(\sigma+$ or $\sigma-)$. The observed PC polarization degree is even higher than the PL polarization since we are able to resolve purely the CPGEdependent valley current. The findings in this work-shed light into the intrinsic photogalvanic effects and helicity-selective transitions in monolayer transition metal dichalcogenides. Besides their fundamental importance, we believe these results may pave the way for application of opto-valleytronic device concepts in interdisciplinary research spanning from nanoelectronics and photonics to biotechnologies.

\section{Methods}

Sample growth and device processing. Monolayer $\mathrm{MoS}_{2}$ flake with size $\sim 5 \mu \mathrm{m}$ is grown by a CVD method (as explained in detail elsewhere ${ }^{24}$ ) onto $\mathrm{Si}$ substrate with $300-\mathrm{nm} \mathrm{SiO}_{2}$. The triangular flake was picked among homogeneous, high crystalline quality flakes then processed into a device, by e-beam lithography with $\mathrm{Ti}(5 \mathrm{~nm}) / \mathrm{Au}(75 \mathrm{~nm})$ contacts for electrical and PC measurements.

PC measurements. By using lock-in technique with a low noise current preamplifier, PC amplitude and phase have been measured for monolayer $\mathrm{MoS}_{2}$ phototransistors at room temperature. The linearly polarized laser beam is centred on the sample with an oblique angle $\theta$ after being chopped to a frequency of $137 \mathrm{~Hz}$, which is the frequency of the measured current at the same time. As shown in Fig. 4, the PC setup has the option to circularly polarize the linearly polarized light field by using a QWP. The angle of photon polarization, $\varphi$, can be varied by rotating the QWP.

\section{References}

1. Novoselov, K. S. et al. Electric field effect in atomically thin carbon films. Science 306, 666-669 (2004)

2. Duan, X., Fu, T.-M., Liu, J. \& Lieber, C. M. Nanoelectronics-biology frontier: from nanoscopic probes for action potential recording in live cells to three-dimensional cyborg tissues. Nano Today 8, 351-373 (2013).

3. Wang, Q. H., Kalantar-Zadeh, K., Kis, A., Coleman, J. N. \& Strano, M. S Electronics and optoelectronics of two-dimensional transition metal dichalcogenides. Nat. Nanotechnol. 7, 699-712 (2012).

4. Chhowalla, M. et al. The chemistry of two-dimensional layered transition metal dichalcogenide nanosheets. Nat. Chem. 5, 263-275 (2013).

5. Hajiyev, P., Cong, C., Qiu, C. \& Yu, T. Contrast and Raman spectroscopy study of single- and few-layered charge density wave material: $2 \mathrm{H}$ - TaSe2. Sci. Rep. 3, 2593 (2013).

6. Wang, Y., Cong, C., Qiu, C. \& Yu, T. Raman spectroscopy study of lattice vibration and crystallographic orientation of monolayer MoS2 under uniaxial strain. Small 9, 2857-2861 (2013).

7. Peimyoo, N. et al. Thernal conductivity determination of suspended mono- and bilayer WS2 by Raman spectroscopy. Nano Res 8, 1210-1221 (2014).

8. Peimyoo, N. et al. Chemically driven tunable light emission of charged and neutral excitons in monolayer WS2. ACS Nano 8, 11320-11329 (2014).

9. Cao, B. C. et al. Low temperature photoresponse of monolayer tungsten disulphide. APL Mater. 2, 116101 (2014).

10. Xiao, D., Liu, G.-B., Feng, W., Xu, X. \& Yao, W. Coupled spin and valley physics in monolayers of MoS2 and other group VI dichalcogenides. Phys. Rev. Lett. 108, 196802 (2012).

11. Mak, K. F., He, K., Shan, J. \& Heinz, T. Control of valley polarization in monolayer MoS2 by optical helicity. Nat. Nanotechnol. 7, 494-498 (2012).

12. Zeng, H., Dai, J., Yao, W., Xiao, D. \& Cui, X. Valley polarization in MoS2 monolayers by optical pumping. Nat. Nanotechnol. 7, 490-493 (2012).

13. Klots, A. R. et al. Probing excitonic states in suspended two-dimensional semiconductors by photocurrent spectroscopy. Sci. Rep. 4, 6608 (2014).

14. Glazov, M. M. \& Ganichev, S. D. High frequency electric field induced nonlinear effects in graphene. Phys. Rep. 535, 101-138 (2014).

15. Mak, K. F., Lee, C., Hone, J., Shan, J. \& Heinz, T. Atomically thin MoS2: a new direct-gap semiconductor. Phys. Rev. Lett. 105, 136805 (2010).

16. Splendiani, A. et al. Emerging photoluminescnece in monolayer MoS2. Nano Lett. 10, 1271-1275 (2010).

17. Kim, S. et al. High-mobility an low-power thin-film transistors based on multilayer MoS2 crystals. Nat. Commun. 3, 1011 (2012).

18. Lopez-Sanchez, O., Lembke, D., Kayci, M., Radenovic, A. \& Kis, A Ultrasensitive photodetectors based on monolayer MoS2. Nat. Nanotechnol. 8, 497-501 (2013).

19. Zhang, Y. et al. Controlled growth of high-quality monolayer WS2 layers on sapphire and imaging its grain boudry. ACS Nano 7, 8963-8971 (2013).

20. Ji, Q. et al. Epitaxial monolayer MoS2 on mica with novel photoluminescence. Nano Lett. 13, 3870-3877 (2013) 
21. Elias, A. L. et al. Controlled synthesis and transfer of large-area WS2 sheets: from single layers to few layers. ACS Nano 7, 5235-5242 (2013).

22. Cong, C. et al. Synthesis and optical properties of large-area single-crysatlline 2D semiconductor WS2 monolayer from chemical vapor deposition. Adv. Opt. Mater. 2, 131-136 (2014).

23. Perea-López, N. et al. Photosensor device based on few-layered WS2 films. $A d v$. Funct. Mater. 23, 5511-5517 (2013).

24. Peimyoo, N. et al. Nonblinking, intense two-dimensional light emitter: monolayer WS2 triangles. ACS Nano 7, 10985-10994 (2013).

25. Yuan, H. et al. Generation and electric control of spin-coupled circular photogalvanic current in WSe2. Nat. Nanotechnol. 9, 851-857 (2014).

26. Xiao, D., Chang, M.-C. \& Niu, Q. Berry phase effects on electronic properties. Rev. Mod. Phys. 82, 1959-2007 (2010).

27. Ghatak, S., Pal, A. N. \& Ghosh, A. Nature of electronic states in atomically thin MoS2 field-effect transistors. ACS Nano 5, 7707-7712 (2011).

28. Yan, J. et al. Dual-gated bilayer graphene hot-electron bolometer. Nat. Nanotechnol. 7, 472-478 (2012).

29. Freitag, M., Low, T., Xia, F. \& Avouris, P. Photoconductivity of biased graphene. Nat. Photon. 7, 53-59 (2013).

30. Buscema, M. et al. Large and tunable photothermoelectric effect in single-layer MoS2. Nano Lett. 13, 358-363 (2013).

31. Tanner, C. et al. Magneto-optic effects in ferromagnetic films: implications for spin devices. Opt. Commun. 259, 704-709 (2006).

32. Renucci, P. et al. Spin-polarized electroluminescence and spin dependent photocurrent in hybrid semiconductor/ferromagnetic heterostructures: an asymmetric problem. Phys. Rev. B 82, 195317 (2010).

33. Villas-Bôas, J., Ulloa, S. E. \& Govorov, A. Spin polarized photocurrent from quantum dots. Phys. Rev. B 75, 155334 (2007).

34. Mak, K. F., McGill, K. L., Park, J. \& McEuen, P. L. The valley Hall effect in MoS2 transistors. Science 344, 1489-1492 (2014).

35. Gong, Z. et al. Magnetoelectric effects and valley-controlled spin quantum gates in transition metal dichalcogenide bilayers. Nat. Commun. 4, 2053 (2013)

36. Avsar, A. et al. Spin-orbit proximity effect in graphene. Nat. Commun. 5, 4875 (2014).

37. Zhu, W. et al. Electronic transport and device prospects of monolayer molybdenum disulphide grown by chemical vapour deposition. Nat. Commun. 5, 3087 (2014).

38. McIver, J. W., Hsieh, D., Steinberg, H., Jarillo-Herrero, P. \& Gedik, N. Control over topological insulator photocurrents with light polarization. Nat. Nanotechnol. 7, 96-100 (2012).

39. Garab, G. \& van Amerongen, H. Linear dichroism and circular dichroism in photosynthesis research. Photosynth. Res. 101, 135-146 (2009).

40. Lynch, I. \& Dawson, K. A. Protein-nanoparticle interactions. Nano Today 3, 40-47 (2008).
41. Gussakovsky, E. E. et al. Left- and right-handed LHC II macroaggregates revealed by circularly polarized cholorophyll luminescence. Photosynth. Res. 87, 253-265 (2006).

42. Cedervall, T. et al. Understanding the nanoparticle-protein corona using methods to quantify exchange rates and affinities of proteins for nanoparticles. Proc. Natl Acad. Sci. USA 104, 2050-2055 (2007).

\section{Acknowledgements}

This work is supported in part by the Singapore Ministry of Education under MOE2013-T1-2-235, MOE2012-T2-2-049, the Singapore National Research Foundation under NRF RF Award No. NRFRF2010-07 and the Agency for Science, Technology and Research, SERC PSF grant 1321202101. C.S. acknowledges the support from the Singapore Ministry of Education MOE2011-T3-1-005 and MOE2013-T2-1-044, and Nanyang Technological University (NAP-SUG M4080511).

\section{Author contributions}

T.Y. supervised the project. M.E. and T.Y. designed the experiments. C.S. coordinated the photocurrent experiments. J.S., C.C. and T.Y. synthesized the flakes. X.S. did the device fabrication. M.E., Z.W. and B.C. carried out the electrical measurements and photocurrent experiments. M.E., J.S., C.C. and B.C. analysed the data. M.E., Z.W., C.S., and T.Y. wrote the manuscript. All authors discussed the results and commented on the manuscript.

\section{Additional information}

Supplementary Information accompanies this paper at http://www.nature.com/ naturecommunications

Competing financial interests: The authors declare no competing financial interests.

Reprints and permission information is available online at http://npg.nature.com/ reprintsandpermissions/

How to cite this article: Eginligil, M. et al. Dichroic spin-valley photocurrent in monolayer molybdenum disulphide. Nat. Commun. 6:7636 doi: 10.1038/ncomms8636 (2015)

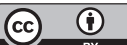

This work is licensed under a Creative Commons Attribution 4.0 International License. The images or other third party material in this article are included in the article's Creative Commons license, unless indicated otherwise in the credit line; if the material is not included under the Creative Commons license, users will need to obtain permission from the license holder to reproduce the material. To view a copy of this license, visit http://creativecommons.org/licenses/by/4.0/ 